\title{
Análise bibliométrica dos temas usabilidade, ambientes virtuais de aprendizagem e
}

\section{pessoas com deficiência}

\section{RESUMO}

Julia Leal Cabral ${ }^{1}$ Thaisi dos Santos Fagundes ${ }^{2}$

Beatriz Lux ${ }^{3}$

Rejane Frozza ${ }^{4}$

A presente pesquisa busca reconhecer as características bibliométricas nos assuntos referentes à usabilidade de sistemas, ambientes virtuais de aprendizagem e pessoas com deficiência, com o objetivo de quantificar a produção na área sobre os respectivos assuntos, com a busca isolada e o cruzamento dos termos. O foco da pesquisa é na usabilidade em ambientes virtuais de aprendizagem e na usabilidade nestes ambientes para pessoas com deficiência. As buscas ocorreram nas bases de dados da Revista Brasileira de Informática na Educação (RBIE), no Simpósio Brasileiro de Informática na Educação (SBIE), na Conferência Internacional sobre Informática na Educação (TISE), no portal de periódicos CAPES (Coordenação de Aperfeiçoamento de Pessoal de Nível Superior) e na base Web of Science. Dentre os resultados das buscas, o baixo número de artigos publicados contendo o cruzamento dos termos definidos pode ser destacado como possível lacuna de conhecimento, indicando a continuidade e avanço das pesquisas nestes assuntos.

Palavras-chave: Usabilidade. Ambientes Virtuais de Aprendizagem. Pessoas com deficiência.

\begin{abstract}
The present research recognizes bibliometric characteristics in areas of usability, virtual learning environments and people with disabilities, aiming to quantify the production area about the respective subject with an isolated search and crossing terms. The point of research consist in results of crossing terms, focused in usability to virtual learning environments and the usability of people with disabilities in these environments. Bibliometric searches occurred in data bases from Revista Brasileira de Informática na Educação, Simpósio Brasileiro de Informática na Educação, Conferência Internacional sobre Informática na Educação, Portal de Periódicos Capes and in Web of Science periodics base. Among search results, it was verified the low number of articles containing the defined crossing terms and none localization in events and journals can be stressed like possible knowledge gaps.
\end{abstract}

Keywords: Usability. Learning Virtual Environment. People with Disabilities.

\footnotetext{
${ }^{1}$ Aluna do Curso de Ciência da Computação da Universidade de Santa Cruz do Sul - UNISC.

${ }^{2}$ Mestranda do Programa de Pós-Graduação em Sistemas e Processos Industriais da Universidade de Santa Cruz do Sul - PPGSPI.

${ }^{3}$ Professora do Departamento de Computação na Universidade de Santa Cruz do Sul.

Professora do Departamento de Computação e do Programa de Pós-Graduação em Sistemas e Processos Industriais na Universidade de Santa Cruz do Sul - PPGSPI. <frozza@unisc.br>
} 


\section{INTRODUÇÃ̃O}

Devido aos avanços das tecnologias da informação e comunicação, a busca de soluções para os problemas encontrados para o seu desenvolvimento e utilização necessita do aporte da produção acadêmica mundial. Assim, evidenciou-se a necessidade de uma busca por materiais na literatura vigente nos temas usabilidade, ambientes virtuais de aprendizagem e pessoas com portadoras de deficiência, e que atendesse aos três temas relacionados.

A base utilizada para a realização da pesquisa bibliográfica foi composta por artigos publicados em eventos e periódicos com relevância na área. As bases de dados escolhidas para a pesquisa foram a Revista Brasileira de Informática na Educação (RBIE), os Anais do Simpósio Brasileiro de Informática na Educação (SBIE), a Conferência Internacional sobre Informática na Educação (TISE), o portal de Periódicos CAPES (Coordenação de Aperfeiçoamento de Pessoal de Nível Superior - Brasil) e a Web of Science. O período de busca de artigos foi de janeiro de 2012 a março de 2015. E quanto aos filtros de localização dos termos pesquisados, as delimitações foram por título e resumo. Assim, essa pesquisa tem como objetivo apresentar os dados quantitativos obtidos na pesquisa realizada, e qualitativos obtidos da análise bibliométrica.

O presente artigo divide-se em seguintes seções: a seção 2 apresenta a fundamentação teórica acerca dos assuntos abordados; a seção 3 aborda os materiais e métodos definidos para a construção da metodologia utilizada na bibliometria; na seção 4 são apresentadas as análises realizadas com os seus respectivos resultados. Por fim, são apresentadas as conclusões do trabalho e as indicações de trabalhos futuros.

\section{FUNDAMENTAÇÃO TEÓRICA}

Na tecnologia, existe uma área específica que trata sobre a ampliação de habilidades da comunicação, sendo esta área denominada Comunicação Alternativa (CA). A Comunicação Alternativa destina-se a pessoas com desabilidades de fala ou que não possuem escrita funcional ou até com defasagem entre necessidades comunicativas de fala e/ou escrita (BERSCH, 2013).

Os evidentes avanços nas tecnologias da informação e comunicação possibilitaram a construção e o desenvolvimento de ambientes flexíveis e adaptados atendendo cada vez mais a individualidade em questões de aprendizado dos estudantes (PASSERINO, 2006). Tendo em 
vista o ganho de forças em relação à inclusão social nas últimas décadas, foram percebidos avanços principalmente na área da educação (BEYER, 2005).

Em questões que apontam a importância da qualificação profissional, a inclusão é evidente e cada vez mais inegável para as empresas. Entretanto, neste caminho, pessoas com deficiência encontram dificuldades em ter acesso à escolaridade, assim comprometendo o seu ingresso no mercado de trabalho (IBDD, 2003). A tecnologia pode ser um recurso que permite acesso a todas as instâncias sociais, tendo o objetivo de incluir e possibilitar o indivíduo exercer a sua autonomia (PEREIRA, 2011).

Com o incremento do número de recursos disponíveis para o aprendizado em ambientes virtuais de aprendizagem, tais como fóruns, chats, atividades de colaboração, questionários, materiais complementares, auxiliado à contínua crescente do uso da internet, é necessária uma abordagem de conceitos, especialmente no que se diz respeito à usabilidade e a adequação destes ambientes para que possam se tornar usuais também a pessoas com deficiência. "O aprendizado se dá não na transferência de conhecimento, mas sim na criação de oportunidades para construção do conhecimento" (FREIRE 1996). Sendo assim, a falta ou o baixo nível de usabilidade em ambientes virtuais de aprendizagem torna-se um dificultador para o aprendizado. Por meio do presente estudo bibliométrico é possível identificar, quantificar e analisar o que vem sendo produzido de material bibliográfico sobre o assunto.

\subsection{Pesquisa Bibliométrica}

A bibliometria surgiu no início do século passado, devido à necessidade de estudar e avaliar as atividades de produção e comunicação científica. É uma "técnica quantitativa e estatística de medição dos índices de produção e disseminação do conhecimento científico" (ARAÚJO, 2006).

Segundo Guedes e Borschiver (2005), bibliometria é "o conjunto de leis e princípios empíricos que contribuem para o estabelecimento dos fundamentos teóricos da Ciência da Informação". Sendo que, o conceito mais utilizado de bibliometria, definido por Pritchard (1969), diz que, "são todos os estudos que tentam quantificar os processos de comunicação escrita".

\subsection{Usabilidade}

Revista Jovens Pesquisadores, Santa Cruz do Sul, v. 6, n. 1, p. 15-30, 2016. 
A usabilidade pode ser definida, conforme a Associação Brasileira de Normas Técnicas-ABNT (2002), como a "capacidade de um produto ser facilmente usado". Sendo que, nos Ambientes Virtuais de Aprendizagem (AVAs) são os usuários que definem qual será o caminho percorrido e como as informações disponibilizadas serão utilizadas. Com isso, busca-se a eficácia do aprendizado em AVAs. Penedo (2012) aborda que a usabilidade visa melhorar a navegabilidade do usuário, assim minimizando os erros e facilitando o seu uso.

A usabilidade deve ser compreendida como a forma de utilizar o ambiente como algo automático, não criando empecilho entre a interface e o usuário, evitando a dispersão durante o usuário navega pelo sistema (ANDRADE, 2007). Pode-se ressaltar que, o uso das tecnologias não está presente apenas para a educação, em Ambientes Virtuais de Aprendizagem, e sim no atual cotidiano (DOURADO, 2015). Contudo, Santos (2008) descreve a usabilidade em sistemas algo que deve ser eficaz, eficiente e agradável nos aspectos que correspondem à interação Homem x Máquina.

Para Nielsen (1993), a verificação de usabilidade possui critérios de análise como: feedback, qualidade e tempo de retorno das ações realizadas pelo usuário, clareza e objetividade na linguagem textual e imagética, consistência na interação com a interface e a sua funcionalidade, facilidade de aprendizagem e memorização do usuário, interface com baixo grau de complexidade, de forma a minimizar a carga cognitiva e facilitando a identificação das ações pelo usuário.

\subsection{Ambientes Virtuais de Aprendizagem}

Os AVAs, cada vez mais estão sendo utilizados no meio acadêmico e corporativo, como uma opção de maior aproximação através de meios tecnológicos às novas técnicas na educação. A partir desse ponto, pode-se observar a necessidade de um entendimento mais aprofundado sobre o conceito do desenvolvimento desses ambientes. Assim, os AVAs são um tipo de estrutura de mescla humana e tecnológica que têm como ponto principal oferecer suporte ao processo de ensino-aprendizagem (PEREIRA, 2007).

No auxílio de um processo de aprendizagem significativo, a estrutura dos AVAs deve possuir diversas mídias com vídeos, áudio, gráficos, textos. Muitos são os recursos disponíveis para permitir uma aprendizagem significativa por meio de AVAs, como: blogs, wikis, chats, fóruns, webteca, midiateca, entre outros. Em AVAs a tecnologia é o meio onde o 
aprendizado ocorre baseado nas táticas pedagógicas utilizadas para obtenção do resultado esperado (PEREIRA, 2007).

Dessa forma, é salutar reconhecer as propriedades pedagógicas que envolvem o desafio de se aprender bem. Em AVAs, tem-se como objetivo fomentar nos estudantes autonomia na aprendizagem, mesmo que essa aprendizagem seja de forma coletiva. Assim, desenvolvendo nos estudantes, habilidades de construção de conhecimento.

Com os novos caminhos que os avanços tecnológicos estão tendo para a educação, é possível atender cada vez mais as individualidades em relação às formas de aprendizado dos estudantes. As variações em questões de técnicas de interface tornam possível a criação de espaços virtuais de aprendizagem que sejam atraentes aos estudantes (KIRNER, 2011) (KIRNER, 2012).

\subsection{Pessoas com deficiências}

A garantia de cidadania plena tendo seus direitos e deveres respeitados é amparada pela Constituição Brasileira pelo Decreto n ${ }^{\circ}$ 3.298, de 20 de dezembro de 1999 (BRASIL, 1999), que caracteriza e assegura às pessoas com deficiência o acesso à educação com os mesmos benefícios conferidos aos demais estudantes. Contudo, a equiparidade em AVAs depende de questões de usabilidade com foco nas deficiências.

O progresso tecnológico contempla a disseminação do conhecimento, e isso inclui pessoas que fazem parte de grupos com deficiências. Dentre inúmeros tipos de deficiência, as deficiências auditiva e visual ainda estão caracterizadas em um grupo segregado, dependendo da atividade a ser desenvolvida e na utilização das tecnologias da informação. Para sanar estas lacunas é possível a utilização de softwares e hardwares específicos, como aporte à respectiva deficiência, desde que o ambiente a ser utilizado esteja acessível a determinadas características (BASSO et al., 2013).

Pessoas com limitações cognitivas podem confrontar-se com inúmeros obstáculos de acesso tanto nas suas atividades escolares quanto nos serviços ofertados de uso comum pela sociedade. De qualquer forma, isso afeta de forma direta nos papéis que ocupam na sociedade. Aplicativos móveis já estão sendo desenvolvidos para atender problemas de acesso a ambientes físicos como shoppings e lojas. Conforme (AUGER et al., 2014) é possível criar um ambiente inclusivo de livre acesso também para pessoas com deficiência. E, com o uso de aplicativos móveis, já foi possível mapear a estrutura de planta de shoppings e lojas, a fim de 
auxiliar na localização de pontos de fácil acesso para cadeirantes, como elevadores e rampas mais próximas.

Após o estudo sobre os temas envolvidos na pesquisa, foi possível aprofundar os conhecimentos e buscar por trabalhos que relacionem estes termos, evidenciando o andamento das pesquisas e a importância atribuída ao desenvolvimento de Ambientes Virtuais de Aprendizagem com usabilidade para pessoas com deficiência.

\section{MATERIAIS E MÉTODOS}

A pesquisa realizada foi baseada na bibliometria e objetivada em uma busca, inicialmente, quantitativa de publicações, e, após, feita a seleção de artigos para uma análise qualitativa. O filtro para período de coleta de dados foi definido de janeiro de 2012 a março de 2015. As bases de dados escolhidas para a realização das buscas foram a revista RBIE, os eventos SBIE e TISE e as bases de periódicos CAPES e Web of Science.

Para o escopo do trabalho foram definidos os termos para busca no título e resumo dos artigos, sendo estes: "Usabilidade", “Ambiente Virtual de Aprendizagem" e "Pessoas com Deficiência”. As pesquisas buscaram cada termo de maneira isolada e na sequência, as relações entre as palavras (intersecções) foram feitas de forma a relacionar dois termos e após os três termos na mesma busca.

Nas bases de periódicos CAPES e Web of Science a busca ocorreu em título e resumo dos artigos, com os termos em inglês: "usability", "virtual learning environment" e "disability"; em espanhol: "comunidad virtual de aprendizage", "usabilidad" e "personas con discapacidad"; e em português: "ambientes virtuais de aprendizagem", "usabilidade" e "pessoas com deficiência". Visando manter a linha de pesquisa do trabalho, foram definidas como áreas de pesquisa a "educação", "ciência da computação" e "engenharias".

Nos anais dos eventos para a busca, a utilização dos termos ocorreu nas línguas espanhola e portuguesa (que são as línguas aceitas pelos referidos eventos para submissão de artigos), sendo que, apesar dos eventos terem edições anuais, a disponibilização dos anais depende de cada evento e não ocorrem no mesmo período. No evento SBIE, encontravam-se disponíveis nos anos de 2012 e 2013, e para o TISE os anais estavam disponíveis nos anos de 2012, 2013 e 2014. 
A pesquisa na Revista Brasileira de Informática na Educação foi realizada com base nos mesmos termos e filtros utilizados nas outras bases. Sendo que, após a busca por termos isolados, foi realizada a busca com as intersecções de dois e três termos.

\section{RESULTADOS}

A Tabela 1 apresenta os resultados da bibliometria quantitativa realizada nas fontes selecionadas, sendo elas: RBIE, SBIE, TISE, CAPES e Web of Science. Os resultados apresentados somam os artigos nas buscas pelos termos em português, inglês e espanhol.

TABELA 1. Levantamento bibliométrico em títulos e resumos.

Quantidade de Artigos Publicados entre janeiro de 2012 e março de 2015, de acordo com os assuntos relacionados na busca por Títulos e Resumos
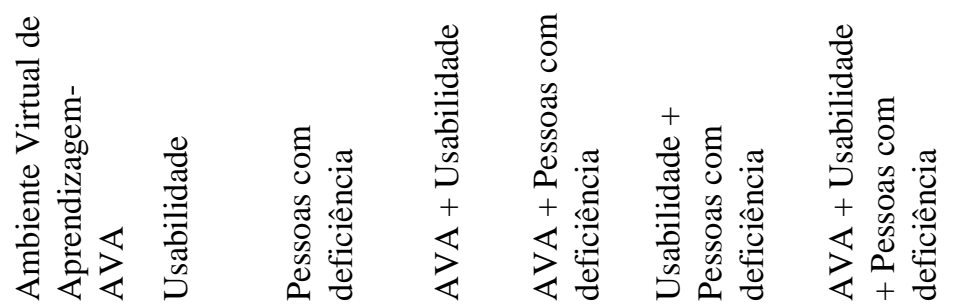

$$
\begin{aligned}
& \text { RBIE - Títulos } \\
& \text { SBIE- Títulos } \\
& \text { TISE - Títulos }
\end{aligned}
$$

$$
12
$$$$
15
$$

3

$$
0
$$

0

0

0

CAPES - Títulos

177

10.843

$$
56 \quad 205
$$$$
1.424
$$

$\begin{array}{ll}0 & 0 \\ 0 & 0\end{array}$

0

0

Web of Science - Títulos

4

RBIE - Resumos

SBIE- Resumos

$21 \quad 13$$$
0 \quad 0
$$$$
0
$$$$
0
$$$$
\begin{gathered}
7 \\
7 \\
0.843
\end{gathered}
$$$$
12
$$$$
5
$$$$
0
$$

TISE - Resumos

25

CAPES - Resumos

226

$1.199 \quad 10.671 \quad 2$$$
1
$$

3

$$
0
$$

Web of Science - Resumos

Fonte: Autoras.

O objetivo do levantamento bibliométrico realizado foi a busca de artigos que contemplem as intersecções entre as palavras-chave. O Gráfico 1 apresenta as intersecções localizadas com dois e três termos em todas as fontes pesquisadas. 


\section{GRÁFICO 1 - Intersecções bibliométricas.}

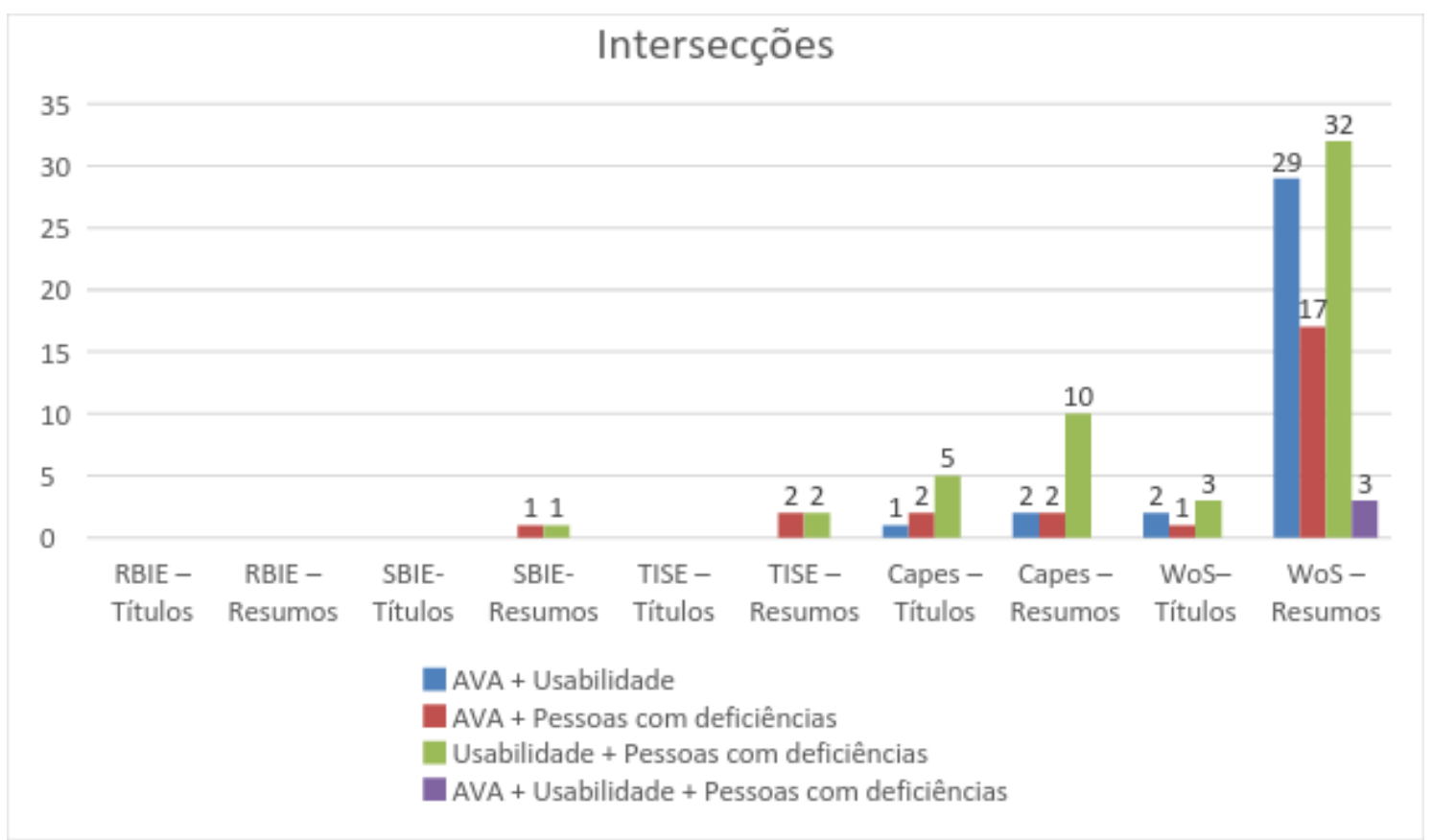

Fonte: Autoras.

O número de intersecções localizadas foi de 112 artigos contendo ao menos dois termos, sendo que destes, três artigos contemplavam os três termos em seus resumos. O maior número de intersecções localizadas foi na base de dados da Web of Science, contando com 6 intersecções de título utilizando o filtro para busca por títulos, e 78 intersecções utilizando o filtro para busca por resumos.

Nos artigos da revista RBIE não foram encontradas intersecções dos termos de busca nos títulos e resumos. Nos eventos, o SBIE apresentou 2 intersecções dos termos de busca nos resumos e o TISE apresentou 4 intersecções dos termos de busca nos resumos. Na base de periódicos da CAPES foram encontradas 8 intersecções dos termos de busca nos títulos e 14 intersecções dos termos de busca nos resumos. Já na base de dados Web of Science foram encontrados os artigos com os três termos de busca contemplando os filtros definidos.

\subsection{Análise qualitativa}

Após esta etapa, foram selecionados artigos encontrados no levantamento quantitativo para realizar a bibliometria qualitativa, que consiste na leitura aprofundada dos artigos e sua análise segundo critérios como: objetivo do trabalho desenvolvido, domínio da 
aplicação, técnicas utilizadas, metodologia da pesquisa, resultados alcançados, proposição de trabalhos futuros e lacunas de pesquisa.

A pesquisa desenvolvida por Mühlbeier e Mozzaquatro (2012), objetivou realizar um estudo aprofundado sobre as dimensões dos estilos de aprendizagem que compõem o modelo do aluno, a fim de desenvolver um sistema para diagnosticar estilos de aprendizagem de estudantes nas modalidades a distância e presencial. A modelagem foi realizada com o uso de diagramas Unified Model Language (UML), uma linguagem destinada a visualizar, especificar, construir e documentar sistemas de software. Os diagramas utilizados são os diagramas de casos de uso e atividades. Em relação aos resultados atingidos, os estilos de aprendizagem de maior predominância foram Estilo Visual, Global, Verbal e Sequencial. Em menor proporção, os estilos Acomodador, Analítico, Assimilador, Reflexivo, Ativo, Convergente, Divergente, Intuitivo, Pessoal, Pragmático, Realista, Sensorial e Teórico. Futuramente, os autores pretendem adaptar o sistema, permitindo o usuário visualizar mais de uma dimensão preferencial; pesquisar métodos e estratégias de aprendizagem para cada uma das dezessete dimensões estudadas; e integrar um módulo ao sistema que permita ao professor visualizar o estilo de aprendizagem de seus estudantes como também indicar materiais instrucionais de acordo com o respectivo estilo. A pesquisa caracterizou-se como descritiva.

O trabalho descrito por Basso, Santorosa e Conforto (2014), objetivou realizar as fases de modelagem e implementação de uma Oficina Multimídia, para criação de materiais nos mais diferentes formatos: histórias, jornais, revistas, poesias, roteiros (teatro, filme, novela) ou histórias em quadrinhos. A ferramenta proposta foi validada juntamente com o AVA Eduquito, utilizando grupos com distintas características (deficientes visuais, com Síndrome de Down e deficientes motores). O protótipo da ferramenta foi desenvolvido com base na plataforma web, o que visa reduzir os requisitos necessários para sua utilização (exigência apenas de computador com um browser e conexão com a internet) e facilitar sua integração a Ambientes Virtuais de Aprendizagem (AVAs). Toda a implementação seguiu as recomendações de acessibilidade, tanto propostas pela WAI/W3C através do Web Content Accessibility Guidelines (WCAG) 2.0, quanto sugeridas por pesquisas ligadas à Informática na Educação Especial. Os resultados de análise da ferramenta frente aos quatro princípios de acessibilidade (perceptível, operável, compreensível e robusto), e de suas respectivas recomendações, apontam para um produto desenvolvido com uma profunda preocupação em respeitar os padrões que favorecem acesso e interação de usuários com as mais distintas capacidades físicas, sensoriais ou intelectuais. A equipe adquiriu experiência nos testes 
realizados durante o desenvolvimento da ferramenta proposta e no seu uso. Há possibilidade de melhorias para um completo atendimento das recomendações da World Wide Web Consortium (W3C). A pesquisa caracterizou-se como descritiva, fazendo uso de fontes bibliográficas para possibilitar a utilização das técnicas utilizadas.

O estudo realizado por Santrosa, Conforto e Basso (2013) teve como objetivo analisar um conjunto de recursos dedicados à abertura de espaços de autoria individual e coletiva no AVA Eduquito, um ambiente virtual implementado em conformidade com os critérios de acessibilidade e usabilidade em todo o seu processo de desenvolvimento e de validação. O processo de validação foi realizado com usuários com diferentes limitações sensoriais, cognitivas e motoras, no Brasil e na Espanha, de uma ferramenta que disponibiliza um espaço para a produção multimídia em diferentes formatos, mesclando linguagens de forma acessível e quadro branco (técnica síncrona). A análise dos aspectos relacionados à simplificação das interfaces e navegabilidade da ferramenta evidenciou alguns problemas nos testes realizados com sujeitos com deficiência visual e também no grupo com Síndrome de Down. Ambos apresentaram dificuldade na operação com as distintas opções de pesquisa de objetos utilizados ou de produções criadas dentro da ferramenta, sendo sugerida a substituição por um sistema de pesquisa simplificada.

Na pesquisa desenvolvida em (AUGER et al., 2014), o objetivo foi identificar as características essenciais em conteúdo e usabilidade de aplicações móveis, a fim de garantir que as necessidades de pessoas com deficiências físicas e de pequenas deficiências em comunicação ou limitações cognitivas fossem atendidas. Um especialista em acessibilidade, a equipe de pesquisa e mais cinco usuários realizaram testes com as aplicações móveis em um shopping center. Este estudo é parte de um projeto maior, desenvolvido no Laboratório de Reabilitação Living in the Mall (RehabMaLL), cujo objetivo é a criação de um ambiente inclusivo em um contexto de shopping para as pessoas que vivem com deficiência. $\mathrm{O}$ desenvolvimento é baseado no âmbito das tecnologias da informação, em uma abordagem de projeto centrado no usuário. É composto de três etapas: (1) definição das necessidades dos usuários para determinar os critérios a serem satisfeitos, (2) testes de usabilidade de forma iterativa, e (3) redefinição de tarefas a serem realizadas pelo aplicativo móvel. Em relação aos resultados descritos, a possibilidade de julgar uma avaliação (por exemplo, útil ou não) foi apreciada, bem como a apresentação dos resultados na forma de um mapa. Foram julgados relevantes os conteúdos abordados na apresentação por quatro tipos de limitações (mobilidade, visão, audição e cognição). No entanto, os usuários não gostavam quando um 
aplicativo impôs um mínimo de pontuação quanto à acessibilidade (por exemplo, nenhuma pontuação abaixo de 5 em uma escala de 10 pontos é permitida). Além disso, um dispositivo móvel (aplicação) que só mostrou a mais recente avaliação não foi considerado como representativa, pois não foi possível ver as opiniões variadas. Descrições curtas e simples de itens foram consideradas, sendo um facilitador para as pessoas com uma pequena limitação na comunicação ou limitação cognitiva. O projeto estabeleceu uma lista de critérios desejados para informar o desenvolvimento de futuras aplicações móveis avaliando as barreiras e facilitadores ambientais que abordam as necessidades do público-alvo. Eventualmente, as avaliações capturadas nessas aplicações móveis podem constituir uma fonte de informação que iria ser continuamente atualizada para medir o impacto das intervenções voltadas para a conscientização da acessibilidade em comerciantes ou para monitorar o impacto das modificações ambientais de shopping centers com a participação de pessoas com deficiência.

Em (SILVA et al., 2014), o objetivo foi criar uma gama de aplicativos móveis para serem utilizados, em sala de aula ou não, a fim de estimular a escrita Braille e o exercício da escrita em Português. O artigo apresentou o projeto, desenvolvimento e avaliação do GBraille Suite, aplicativo móvel composto por uma interface Launcher ("Menu Inicial" do sistema operacional) acessível, um teclado virtual baseado na metáfora Braille e um aplicativo multimodal lúdico para o exercício de ortografia e sinonímia, o GBraille Hangman. A suíte de aplicativos GBraille foi desenvolvida para a plataforma Android e baseia-se em interfaces multimodais, nas quais o uso de um sintetizador de voz é o elemento principal para promover a acessibilidade. O GBraille Suite é composto pelos seguintes aplicativos: GBraille Launcher, uma alternativa de interface acessível a ser exibida ao estudante com deficiência visual no momento em que ele ligar o telefone; GBraille Keyboard, uma solução de entrada de dados em Braille para dispositivos móveis; GBraille Hangman, um aplicativo lúdico educativo no qual os estudantes precisam adivinhar uma palavra, com base em uma informação que é repassada pelo software. É importante ressaltar que alguns elementos visuais estão presentes nessas interfaces, pois podem contribuir na compreensão dos elementos da aplicação no caso de usuários com baixa visão. Alguns resultados listados pelos autores são: i) A interface inicial do GBraille agradou aos usuários e o seu uso tornou-se facilitado graças aos recursos de acessibilidade disponibilizados; ii) Os usuários conseguiram compreender a disposição dos elementos na tela e o que era descrito por meio da síntese de voz; os estudantes estavam satisfeitos com o teclado e com a forma diferenciada de inserção de caracteres; iii) O teclado era utilizado com mais eficiência com o decorrer do tempo e eles gostariam de utilizá-lo 
novamente em outros softwares; iv) Além do melhoramento e enriquecimento da proposta, é fundamental realizar um estudo mais sistemático e profundo da avaliação da usabilidade do sistema e do seu impacto nas aplicações feitas com estudantes na escola para educação especial parceira do projeto.

Na pesquisa de (DIAS et al., 2013), o objetivo focou em avaliar a percepção de respondentes em relação ao uso do modelo Technology Acceptance Model (TAM), a fim de identificar o quanto a colaboração acelera o aprendizado do software Dosvox. A amostra foi composta por 15 estudantes deficientes visuais, matriculados no curso Dosvox. No caso desta pesquisa, a amostra foi organizada tendo como objeto de estudo um software de apoio a pessoas com deficiência visual na modalidade distância, no ambiente virtual de aprendizagem, o software Dosvox. Esta pesquisa verifica como os deficientes visuais reagem à adoção das ferramentas de tecnologia, mais especificamente quanto ao aprendizado do software Dosvox. A pergunta da pesquisa foi formulada da seguinte maneira: "Como ocorre a adoção do Dosvox e a aprendizagem colaborativa entre deficientes visuais em um ambiente virtual de aprendizagem?". O modelo de referência da pesquisa foi o modelo TAM. Quanto aos meios de investigação utilizados, foi conduzida a partir de um survey baseado em questionário. Os autores utilizaram uma metodologia de pesquisa quantitativa através de questionários respondidos pelos estudantes do curso piloto de Dosvox, na modalidade a distância. Os resultados obtidos foram estruturados em três grandes grupos: Análise descritiva dos respondentes, análise dos fatores motivacionais intrínsecos e extrínsecos, e análise de aspectos referentes ao aprendizado colaborativo. A amostra foi formada por homens e mulheres. Dentre os 15 estudantes, apenas 14 destes responderam todo o questionário. Um desses estudantes respondeu apenas as questões de análise descritiva dos respondentes. A análise descritiva da pesquisa mostra que $66,66 \%$ da amostra de participantes são mulheres e $73,30 \%$ dos participantes possuem pós-graduação; 93,3\% dos participantes afirmaram que possuem deficiência visual e 66,6\% destes apresentam esta condição por um período que varia entre 5 e 10 anos; a amostra de participantes da pesquisa utiliza computadores e softwares de acessibilidade por mais de 10 anos, sendo o grau respectivamente para estes de 66,6\% e $60 \%$. Como 93,3\% dos participantes apresentam deficiência visual, o uso de algumas tecnologias assistivas é tarefa comum entre a amostra. Para dar continuidade à pesquisa, os autores relatam aplicação em uma amostra mais significativa, ou seja, no acompanhamento e avaliação de tecnologia assistiva, onde haverá 150 estudantes, muitos deles deficientes visuais 
trabalhando com diversas ferramentas dentro de um ambiente virtual de aprendizagem, ao longo de seis meses.

A tabela 2 apresenta uma comparação dos trabalhos relacionados.

TABELA 2. Comparativo dos trabalhos relacionados.

\begin{tabular}{|c|c|c|c|c|}
\hline Trabalhos relacionados & Objetivo & $\begin{array}{l}\text { Ambiente } \\
\text { (cenário) }\end{array}$ & $\begin{array}{l}\text { Técnicas } \\
\text { utilizadas }\end{array}$ & Método \\
\hline $\begin{array}{l}\text { Mühlbeier e } \\
\text { Mozzaquatro (2012) }\end{array}$ & $\begin{array}{l}\text { Diagnosticar estilos } \\
\text { de aprendizagem de } \\
\text { estudantes }\end{array}$ & $\begin{array}{c}\text { Sistema } \\
\text { desenvolvido }\end{array}$ & $\begin{array}{c}\text { Modelagem } \\
\text { UML, diagramas } \\
\text { de casos de uso e } \\
\text { atividades }\end{array}$ & $\begin{array}{l}\text { Pesquisa } \\
\text { descritiva }\end{array}$ \\
\hline $\begin{array}{l}\text { Basso, Santorosa e } \\
\text { Conforto (2014) }\end{array}$ & $\begin{array}{l}\text { Criar materiais em } \\
\text { diferentes formatos } \\
\text { para Oficina } \\
\text { Multimídia }\end{array}$ & $\begin{array}{l}\text { Ambiente virtual } \\
\text { de aprendizagem } \\
\text { Eduquito }\end{array}$ & $\begin{array}{l}\text { Plataforma web e } \\
\text { AVA, } \\
\text { recomendação } \\
\text { acessibilidade da } \\
\text { WAI/W3C }\end{array}$ & $\begin{array}{c}\text { Pesquisa } \\
\text { descritiva, uso de } \\
\text { fonte bibliográfica }\end{array}$ \\
\hline $\begin{array}{l}\text { Santrosa, Conforto e } \\
\text { Basso (2013) }\end{array}$ & $\begin{array}{c}\text { Analisar recursos } \\
\text { dedicados à } \\
\text { abertura de espaços } \\
\text { de autoria } \\
\text { individual e coletiva }\end{array}$ & $\begin{array}{l}\text { Ambiente virtual } \\
\text { de aprendizagem } \\
\text { Eduquito }\end{array}$ & $\begin{array}{l}\text { Produção } \\
\text { multimídia em } \\
\text { diferentes } \\
\text { formatos, } \\
\text { linguagens de } \\
\text { forma acessível e } \\
\text { quadro branco } \\
\text { (técnica síncrona) }\end{array}$ & $\begin{array}{c}\text { Pesquisa } \\
\text { descritiva e } \\
\text { experimental }\end{array}$ \\
\hline Auger et al. (2014) & $\begin{array}{c}\text { Identificar } \\
\text { características de } \\
\text { usabilidade em } \\
\text { aplicações móveis, } \\
\text { para pessoas com } \\
\text { deficiências físicas }\end{array}$ & $\begin{array}{l}\text { Sistema } \\
\text { desenvolvido } \\
\text { (aplicativo móvel } \\
\text { com uso em } \\
\text { shopping centers) }\end{array}$ & $\begin{array}{l}\text { Uso de } \\
\text { tecnologias da } \\
\text { informação, em } \\
\text { uma abordagem } \\
\text { de projeto } \\
\text { centrado no } \\
\text { usuário }\end{array}$ & $\begin{array}{l}\text { Experimental, uso } \\
\text { do sistema com } \\
\text { pessoas com } \\
\text { deficiência em } \\
\text { shopping centers }\end{array}$ \\
\hline Silva et al. (2014) & $\begin{array}{c}\text { Desenvolver } \\
\text { aplicativos móveis } \\
\text { para estimular a } \\
\text { escrita Braille e o } \\
\text { exercício da escrita } \\
\text { em Português }\end{array}$ & $\begin{array}{c}\text { GBraille Suite } \\
\text { (aplicativo móvel) }\end{array}$ & $\begin{array}{l}\text { Sistema } \\
\text { desenvolvido } \\
\text { (aplicativo móvel } \\
\text { - plataforma } \\
\text { Android) }\end{array}$ & $\begin{array}{l}\text { Experimental, uso } \\
\text { do sistema com } \\
\text { pessoas com } \\
\text { deficiência para } \\
\text { avaliar a } \\
\text { usabilidade }\end{array}$ \\
\hline Dias et al. (2013) & $\begin{array}{c}\text { Analisar e } \\
\text { identificar o quanto } \\
\text { a colaboração } \\
\text { acelera o } \\
\text { aprendizado de } \\
\text { pessoas com } \\
\text { deficiência visual }\end{array}$ & Software Dosvox & $\begin{array}{c}\text { Modelo } \\
\text { Technology } \\
\text { Acceptance Model }\end{array}$ & $\begin{array}{l}\text { Pesquisa } \\
\text { quantitativa, com } \\
\text { uso de } \\
\text { questionários }\end{array}$ \\
\hline
\end{tabular}

Fonte: Autoras.

A partir do estudo dos trabalhos relacionados apresentados, observa-se a importância da participação das pessoas com deficiência na definição das atividades e usabilidade dos sistemas propostos (AVAs, Oficinas, sistemas específicos, entre outros), a fim de respeitar e contribuir para a aprendizagem efetiva deste público-alvo. Os trabalhos, de modo geral, relatam a importância da avaliação dos sistemas, em relação a sua usabilidade e 
funcionalidade; o uso de técnicas que abordem os estilos cognitivos das pessoas com deficiência; a necessidade de estudos mais aprofundados dos AVAs voltados a pessoas com deficiência.

\section{CONCLUSÃO}

Diante do contexto analisado, esse estudo bibliométrico constatou a carência de estudos que supram as necessidades de pessoas que possuem desabilidades visuais e auditivas, ainda que este número seja pequeno em comparação ao número de pessoas que não enfrentam problemas para utilizar ferramentas computacionais ao uso comum.

Contudo, a bibliometria mostrou-se um estudo esclarecedor, que aponta vertentes de pesquisas que acrescem e impulsionam o desenvolvimento de possíveis trabalhos futuros, no que se refere ao tema de ambientes virtuais de aprendizagem relacionado a pessoas com deficiência e atendendo as questões de usabilidade.

\section{REFERÊNCIAS}

ANDRADE, A. Usabilidade de interfaces Web: avaliação heurística no jornalismo on-line. Editora E-papers. 1. ed. 2007.

ARAÚJO, C. A. Bibliometria: evolução histórica e questões atuais. Em Questão, Porto Alegre, n. 1, v. 12, p. 11-32, jan./jun.2006.

AUGER, C. Et al. Mobile Applications for Participation at the Shopping Mall: Content Analysis and Usability for Persons with Physical Disabilities and Communication or Cognitive Limitations. International Journal of Environmental Research and Public Health, Switzerland, n. 12, v. 11, 2014.

BASSO, L. de O; SANTAROSA, L. M. C.; CONFORTO, D. A interação e apropriação tecnológica de pessoas com deficiência através de ferramenta multimídia acessível. Revista Brasileira de Informática na Educação, n. 03, v. 21, p. 12, mar.2014.

BERSCH, R.; SARTORETTO, M. L. Assitiva: Tecnologia e Educação. Disponível online em $<$ http://www.assistiva.com.br/ca.html >. Acessado em 18 de abril de 2015.

BEYER, H.; Inclusão e Avaliação na escola: de alunos com necessidades especiais. Porto Alegre: Mediação, 2005.

BRASIL. Decreto n. 3.298, de 20 de dezembro de 1999. Regulamenta a Lei no 7.853, de 24 de outubro de 1989, dispõe sobre a Política Nacional para a Integração da Pessoa Portadora de Deficiência, consolida as normas de proteção e dá outras providências. Disponível em: < http://portal.mec.gov.br/seesp/arquivos /pdf/dec3298.pdf >. Acessado em: 22 maio 2015. 
DIAS, A. et al. Tecnologia Assistiva e Aprendizagem Colaborativa: Um survey com deficientes visual em ambiente virtual de aprendizagem a partir do Modelo TAM. Nuevas Ideas en Informática Educativa TISE. 2013.

DOURADO, I. F. et al. Uso das TIC no Ensino de Ciências na Educação Básica: uma Experiência Didática. UNOPAR Científica Ciências Humanas e Educação, dez.2014.

FREIRE, P. Pedagogia da autonomia: saberes necessários à prática educativa. São Paulo: Paz e Terra, 25. ed. 1996.

GUEDES, V.; BORSCHIVER, S. Bibliometria: uma ferramenta estatística para a gestão da informação e do conhecimento, em sistemas deinformação, de comunicação e de avaliação científica e tecnológica. In: CINFORM - Encontro Nacional De Ciência Da Informação, 6. 2005, Salvador. Anais... Salvador: ICI/UFBA, 2005.

IBDD. Sem limite: inclusão de portadores de deficiência no mercado de trabalho. Rio de Janeiro: Editora Senac Rio, 2003.

MÜHLBEIER, A. R. K; MOZZAQUATRO, P. M. Estilos e Estratégias de Aprendizagem Personalizadas a Alunos das Modalidades Presenciais e a Distância. Revista Brasileira de Informática na Educação, Porto Alegre, n. 1, v. 20, 2012.

NBR, ABNT. 9241-11. Requisitos ergonômicos para trabalho de escritório com computadores: Parte 11-Orientação sobre usabilidade. Associação Brasileira De Normas Técnicas. Rio de Janeiro: sn, p. 21, 2002.

NIELSEN, J. Usability Engineering. San Francisco, 1993.

PASSERINO, L., M.; SANTAROSA, L., M., C.; Possibilidades da Mediação Tecnológica na Inclusão Escolar de Autistas. Anais do XXVI Congresso da SBC, 2006.

PENEDO, J. R. et al. Análise de usabilidade de um sistema de ead baseada em modelos markovianos e em taxonomia. In Anais do $23^{\circ}$ Simpósio Brasileiro de Informática na Educação, Rio de Janeiro, nov. 2012.

PEREIRA, A. T. C.; SCHMITT, V.; DIAS, M. R. A. C. Ambientes virtuais de aprendizagem. AVA-Ambientes Virtuais de Aprendizagem em Diferentes Contextos. Rio de Janeiro: Editora Ciência Moderna Ltda, 2007.

PEREIRA, A., C., C. Inclusão de pessoas com deficiência no trabalho e o movimento da cultura organizacional: análise multifacetada de uma organização. 2011. 160f. Dissertação (Programa de Pós-Graduação em Educação - Mestrado) - Universidade Federal do Rio Grande do Sul, Porto Alegre, 2011.

PRITCHARD, A. Statistical bibliography or bibliometrics? Journal of Documentation, [s. 1.], n.4, v. 25, p. 348-349, dez. 1969. 
SANTAROSA, C. M. L.; CONFORTO, D. ; BASSO, L. de O. Ferramentas de autoria e de colaboração: discutindo a acessibilidade e a usabilidade na perspectiva da Web 2.0. Revista Brasileira de Informática na Educação, Porto Alegre, n.1, v. 21, 2013.

SANTOS, R. C., GASSENFERTH, W., \& MACHADO, M. A. S. Systems usability evaluation metrics review. In Global information management strategies. Global Business and Technology Association Conference-GBATA, Espanha-Madri, 2008.

SILVA, A. R. S. et al. Especificação e desenvolvimento de um ambiente educativo móvel para a prática da escrita Braille. III Congresso Brasileiro de Informática na Educação (CBIE) e XXV Simpósio Brasileiro de Informática na Educação (SBIE), 2014.

\section{Como citar este documento:}

CABRAL, Julia Leal et al. Análise bibliométrica dos temas usabilidade, ambientes virtuais de aprendizagem e pessoas com deficiência. Revista Jovens Pesquisadores, Santa Cruz do Sul, v. 6, n. 1, jun. 2016. ISSN 2237-048X. Disponível em: <https://online.unisc.br/seer/index.php/jovenspesquisadores/article/view/7298>. Acesso em: ... doi:http://dx.doi.org/10.17058/rjp.v6i1.7298. 